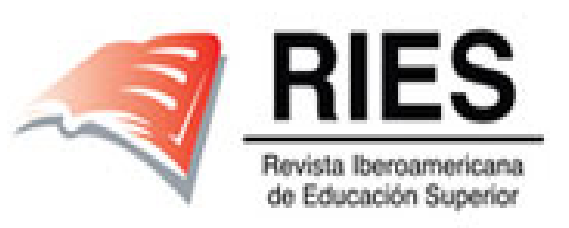

Revista Iberoamericana de Educación Superior

E-ISSN: 2007-2872

emmaro@unam.mx

Instituto de Investigaciones sobre la

Universidad y la Educación

México

Amado-Moreno, María G.; Sevilla-García, Juan J.; Galaz-Fontes, Jesús F.; Brito-Páez, Reyna A. Análisis preliminar de la productividad académica en los institutos públicos tecnológicos mexicanos Revista Iberoamericana de Educación Superior, vol. IV, núm. 11, octubre-enero, 2013, pp. 125-135 Instituto de Investigaciones sobre la Universidad y la Educación .jpg, México

Disponible en: http://www.redalyc.org/articulo.oa?id=299128589007

Cómo citar el artículo

- Número completo

- Más información del artículo

Página de la revista en redalyc.org

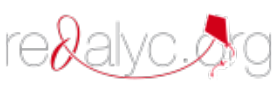

Sistema de Información Científica

Red de Revistas Científicas de América Latina, el Caribe, España y Portugal Proyecto académico sin fines de lucro, desarrollado bajo la iniciativa de acceso abierto 


\title{
Análisis preliminar de la productividad académica en los institutos públicos tecnológicos mexicanos
}

María G. Amado-Moreno, Juan J. Sevilla-García, Jesús F. Galaz-Fontes y Reyna A. Brito-Páez

\begin{abstract}
Resumen
Para conocer las actividades de docencia e investigación que realizan los académicos de los institutos públicos tecnológicos (IPT), se recurre a la información de tres encuestas aplicadas en México en 1992, 2000 y 2007-2008 relacionadas con tres estudios nacionales. En este primer análisis se explora cada una de las bases de datos generadas en estos estudios para conocer cuántos académicos de los institutos públicos tecnológicos participaron en la muestra, el número de grupos atendidos, el porcentaje de tiempo dedicado a docencia e investigación, pertenencia al Programa de Mejoramiento del Profesorado o al Sistema Nacional de Investigadores (SNI). La información preliminar obtenida permite aventurarnos en el conocimiento de la productividad académica en docencia e investigación que han tenido los académicos de estos institutos y proporciona una idea de los cambios que han ocurrido de 1992 a 2008 en estas instituciones públicas de educación superior.
\end{abstract}

Palabras clave: productividad académica, institutos públicos tecnológicos, académico, educación superior, México.

María G. Amado-Moreno

lupitaamado@yahoo.com.mx Mexicana. Maestra en Enseñanza de la Ciencias por el Centro Interdisciplinario de Investigación y Docencia en Educación Técnica (CIIDET), Maestra en Ingeniería Termodinámica por la Universidad Autónoma de Baja California; catedrática de tiempo completo en el Instituto Tecnológico de Mexicali, Departamento de Ciencias Básicas. Temas de investigación: estudio de métodos y estrategias de enseñanza y aprendizaje para ingeniería, los académicos en la educación superior.

Juan J. Sevilla-García

jsevilla@uabc.edu.mx Mexicano. Doctor en Ciencias de la Educación por la Universidad Iberoamericana; Profesor-Investigador de tiempo completo en la Universidad Autónoma de Baja California, Instituto de Ingeniería; miembro del Sistema Nacional de Investigadores. Temas de investigación: estudio de los actores en la educación superior, gestión de la relación universidad-empresa.

Jesús F. Galaz-Fontes

jesusgalaz@uabc.edu.mx Mexicano. Doctor en Educación por Claremont Graduate University, Estados Unidos; Profesor-Investigador de tiempo completo en la Universidad Autónoma de Baja California, Facultad de Ciencias Humanas; coordinador nacional de la Red de Investigadores sobre Académicos, y del proyecto "La reconfiguración de la profesión académica en México"; miembro del Sistema Nacional de Investigadores. Temas de investigación: estudio de los académicos, gobierno universitario en México y a nivel internacional.

Reyna A. Brito-Páez

reynabrito59@gmail.com Mexicana. Maestra en Educación por la Universidad Pedagógica Nacional; Catedrática del Instituto Tecnológico de Mexicali, Departamento de Desarrollo Académico; Coordinadora de Investigación Educativa. Temas de investigación: estudio de métodos y estrategias de enseñanza y aprendizaje para ingeniería, los académicos en la educación superior. 


\title{
Análise preliminar da produtividade acadêmica nos institutos públicos tecnoló- gicos mexicanos
}

\section{Resumo}

Para conhecer as atividades de docência e investigação que realizam os acadêmicos dos institutos públicos tecnológicos (IPT), recorre-se à informação de três pesquisas aplicadas no México em 1992, 2000 e 2007-2008, relacionadas com três estudos nacionais. Nesta primeira análise explora-se cada uma das bases de dados geradas nestes estudos para conhecer quantos acadêmicos dos institutos públicos tecnológicos participaram da amostra, o número de grupos atendidos, a percentagem de tempo dedicado à docência e à investigação, e se pertence ao Programa de Melhoramento do Professorado ou ao Sistema Nacional de Investigadores (SNI). A informação preliminar obtida permite aventurar-nos no conhecimento da produtividade acadêmica em docência e investigação que tiveram os acadêmicos destes institutos, e oferece uma ideia das mudanças que ocorreram entre 1992 e 2008 nestas instituições públicas de ensino superior.

Palavras chave: produtividade acadêmica, institutos públicos tecnológicos, acadêmico, ensino superior, México.

\section{Preliminary analysis of academic productivity in Mexican public technological institutes}

\begin{abstract}
To learn about the teaching and research activities performed by the academicians of public technological institutes (IPT), the information of three surveys performed in Mexico in 1992, 2000 and 2007-2008 related to three national studies is used. This first analysis explores each of the data bases generated in these studies to determine how many academicians of the public technological institutes participated in the sample, the number of groups served, the percentage of time dedicated to teaching and research, membership of the Faculty Improvement Program (Promep) of the National System of Researchers (SNI). The preliminary information allows us to delve into the knowledge of academic productivity in teaching and research of the academicians of said institutes and provides an idea of the changes that occurred from 1992 to 2008 in these public higher education institutions.
\end{abstract}

Key words: academic productivity, public technological institutes, academician, higher education, Mexico. 


\section{Introducción}

El sistema de educación en México es de gran magnitud, diverso, complejo y heterogéneo tanto por las instituciones que lo forman como por las características y perfil de sus académicos. En 2006 estaba formado por 1892 instituciones de educación superior (IES) de las cuales 713 eran instituciones públicas y 1179 privadas (Rubio, 2006).

Los institutos públicos tecnológicos (IPT) son un subsistema del Sistema Nacional de Educación Superior Tecnológica (SNEST) del país, el cual en 2011 atendió a 440116 estudiantes (SNEST, 2012).

Investigar qué hacen los profesores en las IES permite conocer la situación de la profesión académica en México, y el análisis que se presenta tiene como objetivo general conocer la productividad académica, es decir, las actividades de docencia e investigación que realizan los profesores de los IPT del SNEST. Se parte de la información contenida en las bases de datos de tres estudios nacionales realizados sobre académicos en 1992, 2000 y $2007-2008$.

\section{Antecedentes}

El estudio sobre los académicos, actores principales de la vida académica en la educación superior, es tema de reciente investigación. Se cuenta con el estudio realizado en 1992, "Los rasgos de la diversidad: un estudio sobre los académicos mexicanos", que formó parte del Estudio Internacional de la Profesión Académica, realizado por The Carnegie Foundation for the Advancement of Teaching, en el cual estuvo incluido México (Gil et al., 1994). En 2000 se realizó el estudio de "Políticas públicas y cambios en la profesión académica en México en la última década" (Grediaga, Rodríguez y Padilla, 2004) y recientemente, en 2007 y 2008, se realizó el estudio "La reconfiguración de la profesión académica en México" (Galaz y Gil, 2009). Sin embargo, estudios específicos sobre los académicos de los IPT existen pocos: en 2002 Sylvie
Didou Aupetit realizó un estudio sobre "Las políticas de educación superior en los institutos tecnológicos federales"; en 2006 el SNEST publicó el Informe de Rendición de Cuentas 2001-2006, y en 2012 el Anuario Estadístico del SNEST, pero en ambos sólo se menciona brevemente información de los académicos en los institutos públicos tecnológicos, y no se brinda como producto una base de datos con la información de estos académicos. Por ello, para conocer más a estos profesionales, en este trabajo se realiza un análisis preliminar de la productividad académica en estas instituciones.

En este estudio se presenta un análisis descriptivo de los cambios ocurridos en las actividades de docencia e investigación de los académicos de los IPT de 1992 a 2007-2008. Específicamente, se recurre a la información generada en tres encuestas nacionales de los estudios ya mencionados: "Rasgos de la diversidad: un estudio sobre los académicos mexicanos", "Políticas públicas y cambios en la profesión académica en México en la última década" y "La reconfiguración de la profesión académica en México".

\section{La educación superior en México}

Las IES se clasifican en subsistemas como sigue: universidades públicas federales, universidades públicas estatales, institutos públicos tecnológicos, universidades tecnológicas públicas, universidades politécnicas públicas, universidades públicas multiculturales, instituciones para la formación de profesionales, instituciones privadas y centros públicos de investigación (Rubio, 2006).

Los IPT son un subsistema del SNEST e integran al Sistema Nacional de Institutos Tecnológicos. En marzo de 2011, estaba compuesto por 261 institutos públicos tecnológicos federales y estatales o descentralizados, distribuidos en las 32 entidades federativas de la república mexicana, de los cuales 125 son federales y 130 estatales. Se cuenta también con seis centros especializados: 
Centro Nacional de Investigación y Desarrollo Tecnológico (CENIDET), Centro Interdisciplinario de Investigación y Docencia en Educación Técnica (CIIDET) y 4 centros regionales de optimización y desarrollo de equipo (CRODE) (SNEST, 2012).

\section{Estudios sobre académicos en México}

A partir de 1990 se han realizado diversos estudios sobre los académicos en México, se analizan dos de los pioneros en este campo y el más reciente de ellos; para ello se exploran las bases de datos de las tres encuestas nacionales ya mencionadas, mismas que se describen brevemente.

\section{Los rasgos de la diversidad: un estudio} sobre los académicos mexicanos, 1992

La encuesta aplicada formó parte del Proyecto Internacional de la Profesión Académica de The Carnegie Foundation for the Advancement of Teaching en 1992, y la muestra de los académicos se obtuvo aleatoriamente de 20 instituciones de educación superior en México, las cuales se clasificaron en universidades públicas, tecnológicos públicos, instituciones privadas y otras instituciones. La muestra global real fue de 1027 académicos encuestados, de los cuales el $59.3 \%$ eran académicos de tiempo completo. En 1992 no se tenía una idea clara de quiénes eran los que laboraban en las IES en México, y este estudio permitió conocer la distribución de los académicos por tipo de institución a la que pertenecen, género, último grado de estudios, número de grupos atendidos, horas impartidas de cátedra por semana; así como cuántos académicos realizan investigación y cuántos pertenecen al Sistema Nacional de Investigadores (SNI) (Gil et al., 1994). Los resultados de este estudio han contribuido de una manera importante a la consideración de los académicos como objeto de estudio.

\section{Políticas públicas y cambios en la profesión académica en México en la última década, 2000}

Se aplicó la encuesta a una muestra de 3861 académicos de las IES que pertenecían a la Asociación Nacional de Universidades e Instituciones de Educación Superior (ANUIES). Participaron 65 instituciones. La muestra global de académicos encuestados fue 3861 (Grediaga et al., 2004). El estudio aporta información importante sobre los académicos y sus trayectorias desde la perspectiva de las políticas públicas y la influencia que éstas han tenido en la profesión académica en México.

\section{La reconfiguración de la profesión académica en México (RPAM), 2007-2008} La encuesta aplicada formó parte del proyecto internacional The changing academic profession (CAP); se aplicó en el ciclo escolar 2007-2008 a una muestra nacional de académicos de tiempo completo y medio tiempo. La muestra se obtuvo en dos etapas, en la primera se identificaron 101 instituciones de educación superior y, posteriormente, se seleccionaron 2826 académicos de 81 IES que habían proporcionado previamente listados de su personal académico. Finalmente, la muestra global efectiva de académicos encuestados fue de 1973. Las instituciones de donde provinieron los académicos se clasificaron por estratos en centros públicos de investigación, instituciones públicas federales, instituciones públicas estatales, instituciones públicas tecnológicas e instituciones particulares (Galaz y Gil, 2009). Los resultados de este estudio permiten analizar la reconfiguración que ha tenido la profesión académica en México; la encuesta aplicada aporta información sobre las actividades de: carrera y situación profesional, situación general y actividades, docencia, investigación, administración-gestión, información personal y preparación profesional de los académicos. 


\section{Metodología}

En esta investigación se realiza un análisis comparativo de las tres encuestas nacionales sobre académicos en México ya mencionadas, explorando sus bases de datos. Se realizó el análisis para los académicos de tiempo completo, es importante mencionar que los académicos de tres cuartos de tiempo fueron integrados al grupo de los tiempos completos en la encuesta de "Rasgos de la diversidad..." y los académicos exclusivos fueron agregados a los de tiempo completo en la encuesta de "Políticas públicas...". Para obtener la información se utilizó el SPSS (Statistical Package for the Social Sciencies), paquete estadístico que permite el análisis de datos.

Se obtuvo información de la muestra de los institutos públicos tecnológicos en cada una de las encuestas: cantidad, género y habilitación de los académicos encuestados; con respecto a la productividad en docencia, se exploró: el número de grupos atendidos por los académicos, porcentaje de tiempo dedicado a la docencia y horas impartidas de cátedra por semana; para la productividad en investigación: porcentaje de académicos que realizan investigación y los que pertenecen al Programa de Mejoramiento del Profesorado (PROMEP) y al SNI.

\section{Resultados}

En la tabla 1 se observa a los académicos encuestados a nivel global y en los IPT en las tres encuestas nacionales. La encuesta de 2007-2008 reporta mayor porcentaje de encuestados en los IPT a nivel global y de tiempo completo, lo cual es bueno para el objetivo de esta investigación, ya que se contó con información más actual de las actividades que realiza el académico en estas IES.

\section{Tabla 1. Académicos encuestados a nivel global y en los IPT, en tres encuestas nacionales}

\begin{tabular}{|c|c|c|c|c|c|c|}
\hline \multirow{2}{*}{ Encuesta } & \multirow{2}{*}{$\begin{array}{c}\text { Número } \\
\text { total }\end{array}$} & \multicolumn{2}{|c|}{ IPT } & \multirow{2}{*}{$\begin{array}{c}\text { Número } \\
T C^{1}\end{array}$} & \multicolumn{2}{|c|}{ IPT } \\
\hline & & Núm & $\%$ & & Núm & $\%$ \\
\hline RPAM (2007-2008) & 1973 & 315 & 16.0 & 1775 & 281 & 15.8 \\
\hline Políticas Públicas (2000) & 3861 & 223 & 6.0 & 1775 & 108 & 6.0 \\
\hline Rasgos de la diversidad (1992) & 3763 & 102 & 2.7 & 15211 & 78 & 5.1 \\
\hline
\end{tabular}

${ }^{I}$ En esta encuesta los académicos de tres cuartos de tiempo fueron integrados al grupo de los de tiempo completo (TC). 
Es interesante observar en la tabla 2 el aumento de académicas de tiempo completo en los IPT, de $21.2 \%$ en 1992 a $49.6 \%$ en $2007-2008$, así como la disminución de académicos del 78.8\% en 1992 a $41.1 \%$ en $2007-2008$. Las políticas públicas en los IPT han originado que en éstos se impartan carreras humanísticas como: contador público, licenciatura en turismo, arquitectura, así como las asignaturas de desarrollo sustentable, taller de ética, taller de liderazgo, taller de investigación I y II, entre otras, en las cuales la participación de las académicas es notoria.

Tabla 2. Distribución por género de los académicos de TC encuestados en los IPT, en tres encuestas nacionales

\begin{tabular}{|l|c|c|c|}
\hline \multicolumn{1}{|c|}{ Encuesta } & \multirow{2}{*}{ Número } & Masculino & Femenino \\
\cline { 3 - 4 } & & \% & 49.6 \\
\hline RPAM (2007-2008) & 281 & 41.1 & 25.0 \\
\hline Políticas Públicas (2000) & 108 & 74.1 & 21.2 \\
\hline Rasgos de la diversidad (1992) & 85 & 78.8 & \multirow{2}{*}{ \% } \\
\hline
\end{tabular}

En cuanto al grado máximo de estudios, también es notorio el incremento de las maestrías y doctorados, como se observa en la tabla 3. En ocho años se pasó del $23.5 \%$ al $36.1 \%$ y siete años más tarde al $49.6 \%$. Se observa un cambio similar en los estudios de doctorado. Programas como el Estímulo al Desempeño Académico y actualmente el PROMEP, han influido en la formación y actualización del personal académico de los IPT.

Tabla 3. Grado máximo de los académicos de TC de los IPT en tres encuestas nacionales

\begin{tabular}{|l|c|c|c|c|}
\hline \multirow{2}{*}{ Encuesta } & \multirow{2}{*}{ Número } & Licenciatura & Maestría & Doctorado \\
\cline { 3 - 5 } & & $\%$ & $\%$ & $\%$ \\
\hline RPAM (2007-2008) & 281 & 41.1 & 49.6 & 9.3 \\
\hline Políticas Públicas (2000) & 108 & 63.9 & 36.1 & 0.0 \\
\hline Rasgos de la diversidad (1992) & 85 & 75.3 & 23.5 & 1.2 \\
\hline
\end{tabular}


Las horas impartidas de cátedra por semana, el número de grupos atendidos así como el porcentaje de tiempo dedicado a ello, son parte de las actividades de docencia que realiza un académico.

La tabla 4 muestra que en 1992 el 50\% de los académicos en los IPT impartían de 13 a 22 horas de cátedra por semana, ocho años después el 59.3\% 1o hacía. Es importante observar en la tabla 5 que 16 años después de lo reportado por la encuesta "Los rasgos de la diversidad", los académicos imparten un mayor número de horas clase por semana, lo cual implica que, a medida que pasa el tiempo, los académicos dedican más horas a la docencia que a las actividades de investigación y gestión.

Tabla 4. Porcentaje de académicos en los IPT que imparten cátedra por semana

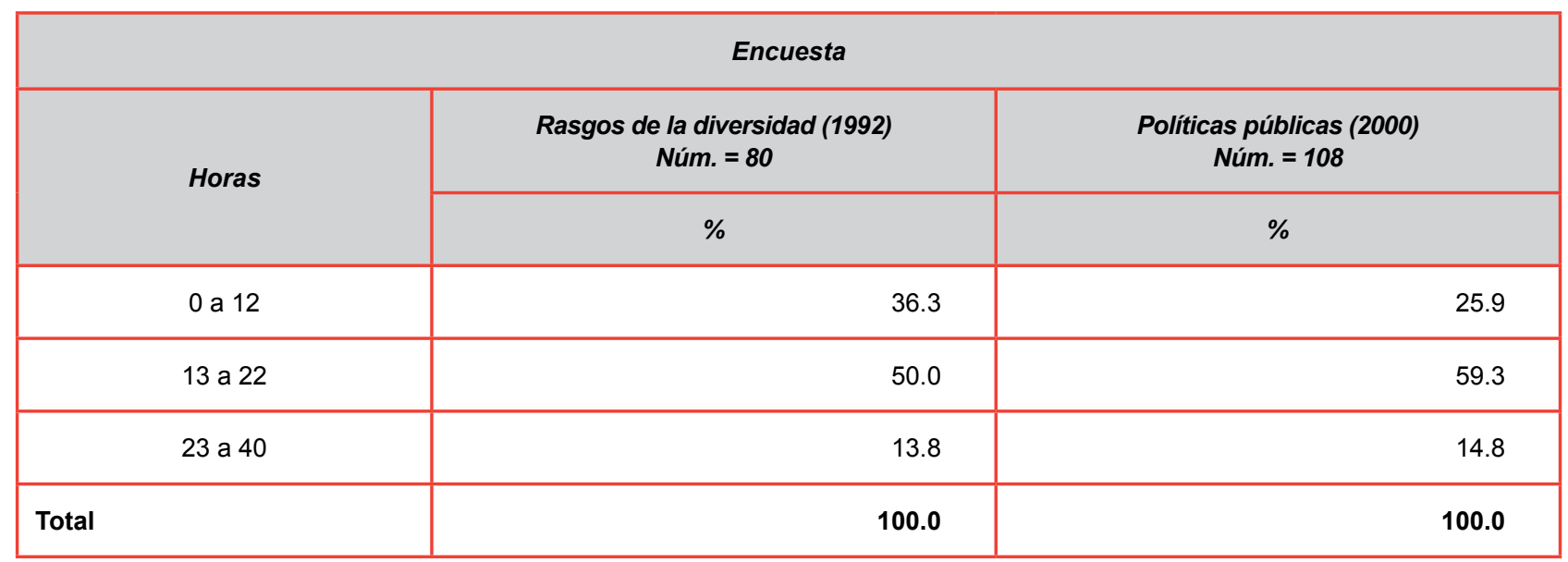

Tabla 5. Porcentaje de académicos en los IPT que imparten cátedra por semana (Núm. = 257)

\begin{tabular}{|c|cc|}
\hline \multirow{2}{*}{ Horas } & \multicolumn{2}{|c|}{ Académicos de TC en los IPT } \\
\cline { 2 - 3 } & \multicolumn{2}{|c|}{$\%$} \\
\hline 0 a 12 & 31.1 \\
\hline 13 a 24 & 65.8 \\
\hline 25 a 36 & Mayor a 36 & 1.2 \\
\hline & & 1.9 \\
\hline Total & & 100.0 \\
\hline
\end{tabular}

Fuente: encuesta RPAM 2007-2008. 
En la tabla 6, los académicos de los IPT reportan que en 2007-2008 el 72\% atendía de uno a cuatro grupos en licenciatura, mientras que sólo el $2.2 \%$ atendía de uno a dos grupos en doctorado. Lo anterior refleja que están más enfocados a atender grupos de licenciatura que de posgrado.

El PROMEP es un programa creado en 1996 por la Secretaría de Educación Pública (SEP) en México para elevar el nivel de habilitación del académico con base en los perfiles de cada subsistema de educación superior; los académicos que acceden a él deben contar con posgrado, realizar actividades de docencia, investigación y gestión. En sus orígenes plantearon transformar el perfil del profesorado en las IES mexicanas en un periodo de diez años (Programa de Mejoramiento del Profesorado, 2012). La encuesta "Los rasgos de la diversidad" no reporta información porque no existía el programa cuando se realizó el estudio y la de "Políticas públicas" no reporta información al respecto. La encuesta RPAM 2007-2008 reporta, como se ve en la tabla 7, que los académicos con PROMEP en los IPT son el 14.9\%, a 16 años de la creación del programa y a cuatro del levantamiento de la encuesta RPAM 2007-2008, el que 15 de 100 académicos reporten pertenecer a este programa no parece suficiente, quizás porque la política pública en cuanto a estrategias de aplicación fue diferente para los IPT que para las universidades (Didou, 2002), ya que este programa se aplicó en los IPT después del año 2000.

Tabla 6. Porcentaje de académicos que atiende grupos en licenciatura y posgrado en los IPT (NLic. = 257, NMaestría = 266, NDoctorado $=269)$

\begin{tabular}{|c|c|c|c|}
\hline \multirow{2}{*}{ Grupos } & \multicolumn{3}{|c|}{ Académicos de TC en los IPT } \\
\cline { 2 - 4 } & \% Licenciatura & \% Maestría & \% Doctorado \\
\hline NO ATIENDE & 1.9 & 84.6 & 2.2 \\
\hline 1 a 2 & 33.5 & 11.3 & 3.0 \\
\hline 3 a 4 & 38.5 & 1.1 & \\
\hline 5 a 6 & 13.6 & & 100.0 \\
\hline 7 a 8 & 9.7 & 100.0 & \\
\hline Mayor que 5 & 2.7 & & 100.0 \\
\hline
\end{tabular}

Fuente: encuesta RPAM 2007-2008.

Tabla 7. Académicos con PROMEP en los IPT

$\left(\mathrm{N}_{\mathrm{T}}=281\right)$

\begin{tabular}{|c|c|c|}
\hline Encuesta & IPT & \% \\
\hline & \% Licenciatura & \% Maestría \\
\hline RPAM (2007-2008) & 42 & 14.9 \\
\hline
\end{tabular}


En las tres encuestas los académicos reportan realizar investigación en los IPT y se observa en la tabla 8 que en 2007-2008 el 34.9\% de los académicos realizaba investigación, mientras que en 1992 solo el $30.6 \%$ lo hacía, un aumento de 4.3 puntos porcentuales en 16 años.

En la tabla 9 se observa que en 2000 el 3.7\% de los académicos pertenecía al SNI y ocho años después sólo aumenta al 5\%, lo que sugiere que los académicos de los IPT no cumplen con los requisitos del Artículo 32 del Diario Oficial de la Federación (2011) que dice: "Para ser miembros del SNI se requiere que los investigadores y tecnólogos realicen habitual y sistemáticamente actividades de investigación científica o tecnológica", además, deben realizar actividades de investigación científica o tecnológica de al menos 20 horas a la semana, lo cual como se ha mostrado no es muy factible, ya que los académicos en los IPT imparten más de 20 horas de cátedra por semana.

Tabla 8. Académicos que realizan investigación en los IPT, en tres encuestas nacionales

\begin{tabular}{|l|c|c|c|}
\hline \multirow{2}{*}{ Encuesta } & Número & \multicolumn{2}{c|}{ Realizan investigación } \\
\cline { 3 - 4 } & TC & Número & 34.9 \\
\hline RPAM (2007-2008) & & 98 & 22.2 \\
\hline Políticas Públicas (2000) & 281 & 24 & 30.6 \\
\hline Rasgos de la diversidad (1992) & 108 & 26 & 2 \\
\hline
\end{tabular}

Tabla 9. Académicos de los IPT en el SNI y nivel, en tres encuestas nacionales

\begin{tabular}{|c|c|c|c|c|c|c|c|c|}
\hline \multirow{3}{*}{ Encuesta } & \multirow{3}{*}{$\begin{array}{c}\text { Número } \\
\text { TC }\end{array}$} & \multirow{2}{*}{\multicolumn{2}{|c|}{ SNI }} & \multirow{3}{*}{$\begin{array}{l}\text { Núm. } \\
\text { SNI }\end{array}$} & \multicolumn{4}{|c|}{ Nivel SNI } \\
\hline & & & & & \multicolumn{2}{|c|}{ Candidato } & \multicolumn{2}{|c|}{ Nivel 1} \\
\hline & & Núm. & $\%$ & & Núm. & $\%$ & Núm. & $\%$ \\
\hline RPAM (2007-2008) & 281 & 14 & 5.0 & 13 & 3 & 23.1 & 10 & 76.9 \\
\hline Políticas Públicas (2000) & 108 & 8 & 3.7 & sd & sd & sd & sd & sd \\
\hline Rasgos de la diversidad (1992) & 78 & 0 & 0.0 & 0 & 0 & 0.0 & 0 & 0.0 \\
\hline
\end{tabular}

$s d=\sin$ datos. 


\section{Tabla 10. Académicos de los IPT que pertenecen a un Cuerpo Académico y el nivel del mismo}

\begin{tabular}{|c|c|c|c|c|c|c|c|c|}
\hline \multirow{2}{*}{ Encuesta } & \multirow{2}{*}{ Número } & \multirow{2}{*}{$\%$} & \multicolumn{2}{|c|}{ En formación } & \multicolumn{2}{|c|}{ En consolidación } & \multicolumn{2}{|c|}{ Consolidado } \\
\hline & & & Núm. & $\%$ & Núm. & $\%$ & Núm. & $\%$ \\
\hline RPAM (2007-2008) & 28 & 10.9 & 8 & 33.3 & 15 & 62.5 & 1 & 4.2 \\
\hline
\end{tabular}

Los cuerpos académicos (CA) son grupos de profesores de tiempo completo con objetivos comunes y que trabajan en una o varias líneas de investigación aplicada o de desarrollo tecnológico (Programa de Mejoramiento del Profesorado, 2012) y es la encuesta RPAM 2007-2008 la que proporciona información sobre la pertenencia de los académicos de los IPT a un cuerpo académico y el nivel de consolidación del mismo. En la tabla 10 se observa que el $10.9 \%$ de los académicos reportan pertenecer a un CA y el $62.5 \%$ de los mismos está en un grupo en consolidación.

\section{A manera de conclusión}

El análisis preliminar de las tres bases de datos de los estudios sobre académicos en México, permite aventurarnos en el conocimiento de la productividad académica en docencia e investigación que han tenido los académicos en los institutos públicos tecnológicos y hoy se tiene una idea de los cambios que han ocurrido de 1992 a 2007-2008 en estas instituciones.

Dentro de los principales hallazgos realizados hasta ahora, está el aumento de las mujeres como académicas de tiempo completo en los IPT, así como que la actualización y formación de los académicos se ve reflejada en las cifras del grado máximo de estudios. Se ha incrementado el porcentaje de académicos de tiempo completo con posgrado en el transcurso de 16 años, acercándose al $50 \%$ los que poseen el grado de maestría.
El análisis de los datos de las tres encuestas reporta que el académico de los IPT privilegia más la docencia, en 1992 el 36.3\% de los académicos impartían menos de 12 horas de cátedra por semana, mientras que en 2007-2008 el 65.8\% impartía de 13 a 24 horas, lo que implica un cambio importante; parece ser que actualmente el académico está más frente a grupo que hace 16 años, ya que imparte muchas horas de clase por semana y atiende de uno a cuatro grupos en licenciatura, y no pueden dedicar 20 horas por semana a la investigación científica o tecnológica, como establece el Reglamento del SNI para ingresar a este programa; por ello, la investigación queda de lado o rezagada en estas IES.

El análisis comparativo de la productividad académica de 1992 a 2008 perfila en términos generales al académico en los institutos públicos tecnológicos, muy centrado en la enseñanza de licenciatura, imparten muchas horas de cátedra y atienden hasta cuatro grupos de licenciatura.

Sin embargo, ésta es una investigación preliminar, sobre todo porque en algunas comparaciones los números de casos implicados son pequeños, por lo que se analizará con más detalle la información de la base de datos del estudio "La reconfiguración de la profesión académica en México" para conocer la productividad en docencia e investigación de los académicos en los IPT mexicanos y confirmar o rechazar este perfil preliminar. Así mismo, se necesitan estudios que permitan contar con muestras más amplias de académicos. 
Se considera que la información que se obtendrá podrá servir para la formulación de políticas, revisar la misión de los IPT, facilitar la toma de decisiones a los directivos, e inclusive señalar el camino para cumplir con las actuales políticas públicas en educación en este subsistema tan diverso y complejo.

\section{Referencias}

Diario Oficial de la Federación (2011), Reglamento del Sistema Nacional de Investigadores, Primera sección, México, Consejo Nacional de Ciencia y Tecnología, 25 de julio de 2011, http://diariooficial.segob.gob. mx [consulta: agosto de 2012].

Didou Aupetit, S. (2002), "Las políticas de educación superior en los institutos tecnológicos federales: una reforma inconclusa", en Revista Mexicana de Investigación Educativa, vol. 7, núm. 14, pp. 51-73.

Galaz Fontes, J. F. y M. Gil Antón (2009), "La profesión académica en México: un oficio en proceso de reconfiguración", en Revista Electrónica de Investigación Educativa, vol. 11, núm. 2, http:// redie.uabc.mx/vol11no2/contenido-galaz2.html [consulta: abril de 2012].

Gil Antón, M. y colaboradores (1994), Los rasgos de la diversidad: un estudio sobre los académicos mexicanos, México, Universidad Autónoma Metropolitana
Azcapotzalco/Casa abierta al tiempo.

Grediaga Kuri, R., J. R. Rodríguez Jiménez y L. E. Padilla González (2004), Politicas públicas y cambios en la profesión académica en México en la última década, México, Asociación Nacional de Universidad e Instituciones de Educación Superior/Universidad Autónoma Metropolitana.

Programa de Mejoramiento del Profesorado (2012), Informe ejecutivo, http://promep.sep.gob.mx/ [consulta: mayo de 2012].

Rubio Oca, J. (coord.) (2006), La politica educativa y la educación superior en México, 1995-2006: un balance, México, Secretaría de Educación Pública/Fondo de Cultura Económica.

Sistema Nacional de Educación Superior Tecnológica (SNEST) (2012), Anuario estadístico 2012, México, SEP-SNEST-DGET, www.dgest.gob.mx [consulta: mayo de 2012].

\section{Cómo citar este artículo:}

Amado-Moreno, María G., Juan J. Sevilla-García, Jesús F. Galaz-Fontes, Reyna A. Brito-Páez (2013), "Análisis preliminar de la productividad académica en los institutos públicos tecnológicos mexicanos", en Revista Iberoamericana de Educación Superior (RIES), México, UNAM-IISUE/Universia, vol. IV, núm. 11, pp. 125-135, http://ries.universia.net/ index.php/ries/article/view/295 [consulta: fecha de última consulta]. 